\title{
RESEARCH ON DATA PROCESSING AND INFORMATION EXTRACTION OF REMOTE SENSING IMAGE BASED ON THE UAV AERIAL PHOTOGRAPHY
}

\author{
Lei Cao \\ First line School of Education, China West Normal University, Nanchong City, Sichuan Province, 637000, China. \\ caolcwnu@126.com
}

\begin{abstract}
Remote sensing technology based on the unmanned aerial vehicle (UAV) aerial photography has high application values in small area monitoring. In this study, a certain village of Nanchong city in Sichuan, China, was taken as the experimental area, the UAV remote sensing technology was used to obtain the image of the area. First of all, image preprocessing was conducted. Then different scales was selected according to the object to segment images, the optimal segmentation scale was selected, and the segmentation hierarchy table was established. The classification rules were defined at each level according to the object difference, and the object was extracted to realize the information extraction of land use situation. It was found from the classification accuracy evaluation that the overall accuracy of the information extraction method in this study was $92.47 \%$ and the Kappa coefficient was 0.91 , which indicated the good quality of the classification; therefore the method was effective. It indicated that the remote sensing technology and object-oriented multi-scale segmentation method can effectively extract information.
\end{abstract}

Keywords: UAV, Remote Sensing, Information Extraction, Object-Oriented, Multi-Scale Segmentation, Classification Accuracy.

\section{Introduction}

The application of unmanned aerial vehicle (UAV) in acquisition of remote sensing image is a new technology, which has practical values in many fields. UAV is not only cheaper but also easier to operate [1]. UAV remote sensing technology has good practicability in land use and topographic mapping, especially in agriculture [2]. Tian et al. [3] acquired the images of wheat and corn in a region by UAV remote sensing technology and proposed an automatic classification method. The reflectance of green band and infrared band was compared to classify wheat, light soil and shadow soil roughly, and then normalized differential vegetation index (NDVI) was calculated for further classification. The classification results indicated that this classification method has an accuracy of $96.18 \%$ in wheat identification and $90.14 \%$ in corn identification, which proved the reliability of the method. Jin et al. [4] classified the crops by UAV remote sensing technology and adopted the object-based method. Segmentation parameters, scale, shape, color, consistency and flatness, were set as 250, 0.1, 0.9, 0.5 and 0.5 respectively. The results showed that the overall accuracy of classification was $85.0 \%$ and the kappa coefficient was 0.82 , which indicated that this technology can be widely applied in data acquisition in agricultural sector. Guo et al. [5] extracted crop types from some farmland in the Eighth Division of Xinjiang Corps and analyzed the color and texture information of different crops to distinguish different crop types based on the visible light image of UAV remote sensing. The results showed that crop classification methods based on color and texture features had higher accuracy and are worthy of promotion. In the disaster information processing, the UAV remote sensing technology also has a good effect. In the case of sudden disasters, it is very important to obtain disaster information quickly and accurately and UAV remote sensing technology has great advantages in this respect [6]. In this study, a village of Nanchong city in Sichuan, China, was taken as an example, the land use in this region was analyzed through UAV remote sensing technology, and different land types was classified by objectoriented multi-scale segmentation method after obtaining images, so as to understand the effectiveness of this method in information extraction.

\section{The UAV Remote Sensing Technology}

Unmanned aerial vehicles (UAV) are vehicles that are controlled remotely by radio or computer. They are simple in structure, cheap in cost, easy to 
maintain and of high application value. UAV remote sensing technology refers to the combination of UAV technology and remote sensing technology. UAV is taken as the aerial platform to obtain information through remote sensing sensor, and then computer is used to process the acquired image information. $\mathrm{UAV}$ is of great application value in the acquisition of land resources, natural resources, disaster area environment and other aspects.

With the development of economy, the landform of different regions in China is changing constantly, which puts forward new requirements for remote sensing technology.

As a new application technology, UAV remote sensing technology is simple in operation, less restricted by environment, and has higher image resolution and faster acquisition speed. It is widely applied in resource monitoring, environmental monitoring, water and soil utilization monitoring, etc.

\section{Data Acquisition and Processing}

In this study, a village of Nanchong city in Sichuan province was taken as an experimental area, and information of land use in that area was extracted. LT - 150 type oil jet (Table 1), Canon 5 d Mark II camera (Table 2) and $24 \mathrm{~mm}$ focal length lens were used.

Table 1. The parameters of UAV

\begin{tabular}{|l|l|l|l|}
\hline $\begin{array}{l}\text { Length of } \\
\text { aircraft }\end{array}$ & $2.2 \mathrm{~m}$ & Load & $10 \mathrm{~kg}$ \\
\hline Duration & $2.5 \mathrm{~h}$ & Flight speee & $\begin{array}{l}80- \\
120 \mathrm{~km} / \mathrm{h}\end{array}$ \\
\hline $\begin{array}{l}\text { Measurement } \\
\text { radius }\end{array}$ & $20 \mathrm{~km}$ & $\begin{array}{l}\text { Wind } \\
\text { resistance }\end{array}$ & $\begin{array}{l}\text { Below } \\
\text { level } 4\end{array}$ \\
\hline
\end{tabular}

Table 2. The parameters of camera

\begin{tabular}{|l|l|l|l|}
\hline Sensor & $\begin{array}{l}\text { Canon 5D } \\
\text { Mark II }\end{array}$ & $\begin{array}{l}\text { Focal } \\
\text { length }\end{array}$ & $\begin{array}{l}25 \mathrm{~mm} / 35 \\
\mathrm{~mm}\end{array}$ \\
\hline Pixel & $\begin{array}{l}21 \text { million, } \\
5616^{*} 3744\end{array}$ & $\begin{array}{l}\text { Single } \\
\text { image } \\
\text { size }\end{array}$ & $<10 \mathrm{MB}$ \\
\hline
\end{tabular}

The relative flight height of the UAV was $1000 \mathrm{~m}$, the ground resolution was $0.27 \mathrm{~m}$, the course overlap degree was $80 \%$, and the side overlap degree was $60 \%$.

The original image obtained is shown in Figure 1.

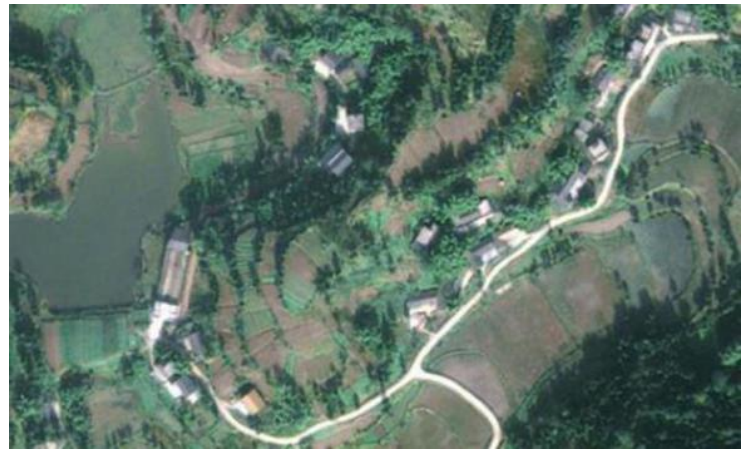

Figure 1: UVA image

For image preprocessing of the obtained image, the correction of UAV image was conducted firstly [7]. The edge parts was cut, and histogram matching method was applied to make uniform color processing. Then the aerial triangulation was conducted. The coordinate of the corresponding storefront discrete point was calculated, digital elevation model (DEM) was generated, and images was quickly generated through orthogonal projection production software [8, 9]. Image segmentation and information extraction were performed in the next step.

\section{Multi-scale Image Segmentation Method}

Image segmentation refers to segmenting an image into several regions with features that do not overlap each other, and the segmented region is the image region. Before segmentation, the principle of segmentation need to be determined. In the land use, the image color, texture and shape of arable land, roads, houses, woodland and water are all different, and the division principle can be established according to the different characteristics. Multi-scale image segmentation method was selected in this study.

The multi-scale segmentation method [10] is to obtain the results with a hierarchical structure by segmenting an image multiple times using a hierarchical segmentation strategy. Each new segmentation is based on the previous segmentation. The segmentation scale of image segmentation will affect the accuracy of image classification. If the segmentation scale is too large, small objects will be hard to be segmented. If the segmentation scale is too small, the same object may be divided into multiple objects, which is not conducive to the subsequent image classification.

The optimal segmentation scale is the peak value of the mean standard deviation of image brightness, which can reflect the type of land use clearly. Experiments have shown that over segmentation will happen if the scale is smaller than 100 and object mixture will happen if the scale is larger than 
350. Therefore, the scale is set between 100 and 350, and the brightness mean standard deviation is calculated to obtain the optimal segmentation scale. The compactness of image is set as 0.5 and the shape factor is 0.6 .

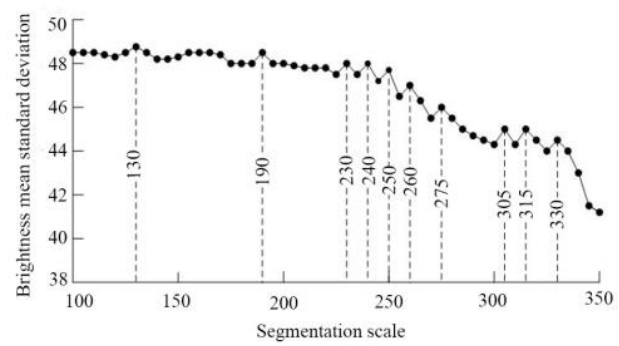

Figure 2: Brightness mean standard deviation under different segmentation scales

Ten optimal segmentation scales can be obtained from the Figure 2. For seven types of land use, including residential land, transportation land, arable land, bare land, paddy fields, woodland and water areas, the four scales of 230, 240, 305 and 315 were selected to establish the segmentation hierarchy table.

Table 3. Multi-scale segmentation hierarchy table

\begin{tabular}{|l|l|l|}
\hline $\begin{array}{l}\text { Segmentation } \\
\text { hierarchy }\end{array}$ & $\begin{array}{l}\text { Segmentation } \\
\text { scale }\end{array}$ & $\begin{array}{l}\text { Segmentation } \\
\text { objects }\end{array}$ \\
\hline L1 & 315 & $\begin{array}{l}\text { Transportation } \\
\text { land and } \\
\text { paddy fields }\end{array}$ \\
\hline L2 & 305 & $\begin{array}{l}\text { Woodland and } \\
\text { water areas }\end{array}$ \\
\hline L3 & 240 & $\begin{array}{l}\text { Arable land } \\
\text { and bare land }\end{array}$ \\
\hline L4 & 230 & $\begin{array}{l}\text { Residential } \\
\text { land }\end{array}$ \\
\hline
\end{tabular}

\section{Object-oriented Multi-scale Segmentation and Information Extraction}

(1) Extract transportation land and paddy fields from L1

In the image, transportation land and some residential land were all white, which can be distinguished from other land types by the brightness mean feature X:

$$
X=\frac{\bar{R}+\bar{G}+\bar{B}}{3}
$$

where $\bar{R}, \bar{G}, \bar{B}$ stands for the average brightness of red, green and blue image elements. Y:

Roads can be distinguish by shape index feature

$$
Y=\frac{e}{4 \sqrt{S}}
$$

where e stands for the boundary length of the object, and $\mathrm{S}$ stands for the object area.

Residential land can be distinguished by gray difference vector mean feature $\mathrm{Z}$ :

$$
Z=\sum_{k=0}^{N-1} k V_{k}
$$

where VK stands for the sum of the diagonal of the gray co-occurrence matrix of the object, and N stands for the number of rows or columns.

The number of adjacent dark objects was set up, and the number of adjacent dark objects was relatively large, so feature 0 was relatively large.

Classification rule 1 was defined as $\mathrm{X}>127, \mathrm{Y}>3.3$, and $0 \geq 5$.

The range of paddy fields was determined by the mean feature $\bar{B}$ of blue wave segment, and the feature $\mathrm{P}$ was established:

$$
P=2 \bar{B}-\bar{R}-\bar{G}
$$

Classification rule 2 was defined as $91<\bar{B}<96$, and $\mathrm{P}>0$.

(2) Extract woodland and water areas from L2

The woodland and water areas were extracted on the basis of L1, the mean feature $\bar{G}[41,77]$ of green band were extracted, and the classification rule 4 was defined as $62<\mathrm{X}<66$, and $\mathrm{P}>0$. The water areas was extracted, the classification rule 5 was defined as $\mathrm{X}>70$, and a small amount of arable land were eliminated.

(3) Extract arable land from L3

Arable land and bare land were extracted on the basis of L2. As the mean values of the red and green bands of the two terrains varied greatly, the classification rule 6 was firstly defined as $70<\mathrm{A}<127$ to extract arable land and bare land simultaneously, and then the features $Q$ and $H$ were established:

$$
\begin{aligned}
& Q=2 \bar{G}-\bar{R}-\bar{B} \\
& H=2 \bar{R}-\bar{B}-\bar{G}
\end{aligned}
$$


Classification rule 7 was defined as $Q \geq 9$, and arable land was extracted. Classification rule 8 was defined as $\mathrm{H}>0$, and $\mathrm{Q}<0$, and bare land was extracted.

(4) Extract residential land from L4

Residential land was extracted on the basis of L3. Residential land include both blue and dark roofs. Firstly, blue roof was extracted with the mean value of blue wave segment, and classification rule 9 was defined as $\bar{B}>158$. Then dark roof was extracted. The mean value of red and blue wave segment of dark roof was greater than that of green band. Classification rule 10 was defined as $74<X<159$, and $\mathrm{Q}<-5$. Through four levels of extraction, the final information extraction results of this region are shown in Figure 3.

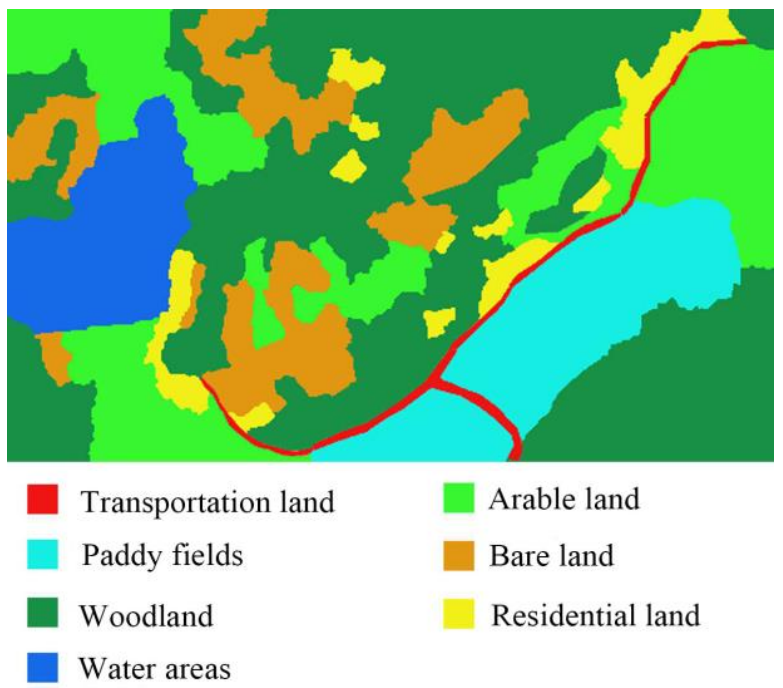

Figure 3: Information extraction results

It can be seen from the information extraction results that woodland area was relatively large, which accounted for about a third of the total area. Arable land concentrated near the water areas and paddy fields, and transportation land was relatively small, indicating that traffic in this area was not very convenient. Residential land was mostly concentrated in the surrounding roads, which was convenient for traveling, and there was some bare land in this region. From the perspective of land use, there was a large amount of land in the region, such as bare land and some woodland, which was worth developing and utilizing to promote the development of the region.

\section{Classification Accuracy Evaluation}

Classification accuracy refers to the accuracy of object extraction, which can be used for testing the validity of classification rules [11]. In order to evaluate the classification accuracy, first of all, the classification results should be roughly evaluated through visual interpretation to determine whether the classification results are roughly consistent with the original image, and then the accuracy test should be carried out further, i.e., the objects after classification are compared with the actual situation. In this study, the confusing matrix method was selected, including three indicators of overall accuracy, overall Kappa coefficient and various types of Kappa coefficient.

(1) Overall accuracy refers to the number of correct classification as a percentage of the total, and its expression is:

$$
P=\frac{\sum_{h=1}^{k} P_{h h}}{M} \times 100 \%
$$

where $P$ stands for the overall accuracy, $P_{h h}$ stands for the correct number of classifications of class $h$, and $\mathrm{M}$ stands for the total number.

(2) Overall Kappa coefficient refers to the degree of coincidence of the two images, and its expression is:

$$
K=\frac{N \sum_{h=1}^{m} P_{h h}-\sum_{h=1}^{m}\left(P_{e h} \times P_{f h}\right)}{N^{2}-\sum_{h=1}^{m}\left(P_{e h} \times P_{f h}\right)}
$$

where $\mathrm{N}$ stands for the total number, $\mathrm{m}$ stands for the total number of classification object types, $P_{e h}$ stands for the total number of columns in each category, $P_{f h}$ stands for the total number of rows in each category, and $P_{h h}$ stands for the number of correct classification of class $h$.

(3) Various types of Kappa coefficient

$$
K_{h}=\frac{N P_{h h}-P_{e h} \times P_{f h}}{N P_{e h}-P_{e h} \times P_{f h}}
$$

The classification accuracy evaluation results in this study are shown in Table 4. 
Research on Data Processing and Information Extraction of Remote Sensing Image Based on the UAV Aerial Photography

Table 4. The classification accuracy evaluation results

\begin{tabular}{|c|c|c|c|c|c|c|c|}
\hline & $\begin{array}{l}\text { The total } \\
\text { number of } \\
\text { samples }\end{array}$ & \begin{tabular}{|c|} 
The total \\
number of \\
classification
\end{tabular} & $\begin{array}{c}\text { The number } \\
\text { of correct } \\
\text { classification }\end{array}$ & $\begin{array}{c}\text { Production } \\
\text { accuracy }\end{array}$ & $\begin{array}{c}\text { User } \\
\text { accuracy }\end{array}$ & $\begin{array}{c}\text { Overall } \\
\text { accuracy }\end{array}$ & $\begin{array}{c}\text { Kappa } \\
\text { coefficient }\end{array}$ \\
\hline $\begin{array}{l}\text { Transportation } \\
\text { land }\end{array}$ & 18 & 19 & 16 & $88.89 \%$ & $84.21 \%$ & \multirow{7}{*}{$92.47 \%$} & \multirow{7}{*}{0.91} \\
\hline Paddy fields & 80 & 78 & 74 & $92.50 \%$ & $94.87 \%$ & & \\
\hline Woodland & 201 & 208 & 169 & $84.08 \%$ & $81.25 \%$ & & \\
\hline Water areas & 59 & 58 & 56 & $94.92 \%$ & $96.55 \%$ & & \\
\hline Arable land & 176 & 178 & 149 & $84.66 \%$ & $83.71 \%$ & & \\
\hline Bare land & 79 & 72 & 64 & $81.01 \%$ & $88.89 \%$ & & \\
\hline Residential land & 52 & 49 & 42 & $80.77 \%$ & $85.71 \%$ & & \\
\hline
\end{tabular}

As can be seen from the Table 4, the number of correct classification of multi-scale classification methods was large, and the classification accuracy rate was high. It was found that the classification accuracy of transportation land, paddy fields and water areas was higher, probably these areas were more concentrated, and it was easy to distinguish, resulting in the small classification error. The distribution of other areas was scattered and difficult to classify, especially arable land, bare land and residential land, resulting in a relatively low accuracy rate. However, from the overall, the overall accuracy of the classification reached $92.47 \%$, and the Kappa coefficient was 0.91 , indicating that the classification quality was relatively high and the effect was relatively good.

\section{Discussion and Conclusion}

With the development of remote sensing technology, its application is becoming more and more widely, and the UAV remote sensing technology has a high application value, especially in the small area monitoring. It can quickly and accurately acquire images of the target area without being restricted by the weather or terrain and has good practicability in resource monitoring, land use, topographic mapping, disaster information processing, etc [12].

In this study, firstly, UAV remote sensing technology was used to obtain the image of the target area, and then the image was preprocessed to improve the accuracy of information extraction. In terms of image segmentation and classification, there are methods based on edge detection, threshold and region, and object-oriented multiscale segmentation method was selected in this study, which can segment the image into a collection of objects with similar features, so as to realize image classification [13]. It is quite important for multi-scale segmentation method to select a good scale. Ten optimal segmentation scales were obtained based on the calculation of a variety of scales, then four scales of 230, 240, 305, 315 was selected from ten optimal segmentation scales for the seven land use types, residential land, transportation land, arable land, bare land, paddy fields, woodland, and water areas, and then the images of them were segmented according to levels. Different classification rules were defined according to the color and shape of different objects to extract different objects. From the extraction results of each level, it could be found that the results of information extraction of the object-oriented multi-scale segmentation method was clear, and the specified object can be extracted explicitly, without overextraction or over-generalization.

In order to further verify the classification quality of this method, the confusing matrix method was selected for classification accuracy evaluation. It was found from calculation that this method had an overall accuracy of $92.47 \%$, and the Kappa coefficient was 0.91 . The classification accuracy suggested that this method had a high classification quality and was effective and reliable in the information extraction of land use.

UAV remote sensing images are of great value in the rapid acquisition of land use information, especially in areas with poor climatic conditions and complicated topography. In this study, the objectoriented multi-scale segmentation method was used, the optimal segmentation scale was selected to segment images according to different levels, and 
information extraction of different objects was achieved. In classification accuracy evaluation, the overall accuracy of this method was $92.47 \%$, and Kappa coefficient was 0.91 , which indicated the high reliability of the method. This study provides a new idea for UAV remote sensing image processing.

\section{Acknowledgement}

This study was supported by Talent Research Fund Project: Research on Interactive Experimental Teaching Platform Based on Augmented Reality (AR) Technology and Its Application in General Technical Courses in Middle Schools (project number: 17YC195).

\section{References}

[1] Azmi S M, Ahmad B, Ahmad A. "Accuracy assessment of topographic mapping using UAV image integrated with satellite images," 2014, 1802-1811.

[2] $\mathrm{Lu} \mathrm{H}, \mathrm{Li} \mathrm{L}, \mathrm{He} \mathrm{Y}$, et al. "Method of UAV Image Mosaic Based on Weighted Adjustment Considering Terrain Feature," Transactions of the Chinese Society for Agricultural Machinery, 2015, 46(9):296-301.

[3] Tian Z K, Fu Y Y, Liu S H, Liu F. "Rapid crops classification based on UAV low-altitude remote sensing," Transactions of the Chinese Society of Agricultural Engineering, 2013, 29(7):109116(8).

[4] Jin K P, Park J H. "Crops Classification Using Imagery of Unmanned Aerial Vehicle (UAV)," Ref, 2015, 57(6):91-97.

[5] Guo P, Wu F D, Dai J G, Wang H J, Xu L P, Zhang G S. "Comparison of farmland crop classification methods based on visible light images of unmanned aerial vehicles," Transactions of the Chinese Society of Agricultural Engineering, 2017, 33(13):112-119.
[6] Li M, Li D, Fan D. "a Study on Automatic Uav Image Mosaic Method for Paroxysmal Disaster," ISPRS - International Archives of the Photogrammetry, Remote Sensing and Spatial Information Sciences, 2012, XXXIX-B6:123-128.

[7] Honkavaara E, Hakala T, Markelin L, et al. "A Process for Radiometric Correction of UAV Image Blocks," Photogrammetrie - Fernerkundung Geoinformation, 2012, 2012(2):117-127.

[8] Liu H, Yuan Z, Zhang J, et al. "Highly efficient paddy classification using UAV-based orthorectified image," IGARSS 2017 - 2017 IEEE International Geoscience and Remote Sensing Symposium. IEEE, 2017:3230-3233.

[9] Park J K, Lee K W. "Classification of Buildings Using High Resolution UAV Image," Green and Smart Technology. 2016:145-147.

[10] Yu H, Wang J, Bai Y, et al. "Analysis of large-scale UAV images using a multi-scale hierarchical representation," Geo-spatial Information Science, 2018(3):1-12.

[11] Lee K D, Lee Y E, Park C W, et al. "A Comparative Study of Image Classification Method to Classify Onion and Garlic Using Unmanned Aerial Vehicle (UAV) Imagery," Korean Journal of Soilence \& Fertilizer, 2016, 49(6):743-750.

[12] Gerke M, Przybilla H. "Accuracy Analysis of Photogrammetric UAV Image Blocks: Influence of Onboard RTK-GNSS and Cross Flight Patterns," Photogrammetrie - Fernerkundung Geoinformation, 2016, 2016(1):17-30.

[13] Zhang X, Chen G, Wang W, et al. “Object-Based Land-Cover Supervised Classification for VeryHigh-Resolution UAV Images Using Stacked Denoising Autoencoders," IEEE Journal of Selected Topics in Applied Earth Observations \& Remote Sensing, 2017, 10(7):3373-3385. 